\title{
Shallow Discussion on Simulation Design of Cleaning Robot Controller Based on Matlab
}

\author{
Li Fangyun, Zhao Wei \\ Institute of Technology, East China Jiaotong University, Nanchang Jiangxi, 330100, China
}

Keywords: Matlab; cleaning robot controller; simulation analysis

\begin{abstract}
Cleaning robot can provide cleaning services, which is greatly needed in the market. Matlab software can be applied to better conduct the simulation of cleaning robot controller, which can solve contradictory problems between requirements of students for the class and actual class environment, and can also help users to better make the design of cleaning robot controller and debugging of problems. This paper include framework and simulation process to analyze the simulation design of cleaning robot controller based on Matlab.

With the continuous development of computer and progress of mechanical manufacturing technology, robot is widely applied in modern market. Cleaning robot can provide cleaning services, which is greatly needed in the market. And the simulation design of cleaning robot controller with Matlab can help relevant personnel to have a clearer understanding of cleaning robot controller.
\end{abstract}

\section{Background}

\subsection{The gap in demand for robot}

With the development of artificial intelligence technology, people are familiar with the concept of robot. Robot can substitute people to get some things done and there is a large gap in the demand for robot in modern society. With the development of social economy, more and more robot enter people's life and will become a future can be prospected. Cleaning robot is a basic robot, and the wide application in people's life is also hopeful, therefore, the simulation analysis of cleaning robot has certain actual social significance.

\subsection{Matlab simulation design}

Matlab software is an excellent software, and provides a large amount of data analysis tools, with strong compatibility. In actual class, not all students can operate robot. Thus, Matlab simulation analysis can better solve the requirements of class. Besides, there is higher stability and accuracy in Matlab simulation analysis of cleaning robot, suitable for application.

\subsection{Cleaning robot controller}

Controller, an important part of cleaning robot, can control robot, deal with external time and give full play the effect of cleaning robot. The stability and performance of cleaning robot, to some extent, are related to controller, which is also the main part of simulation analysis of cleaning robot.

\section{Framework}

In Matlab simulation analysis, cleaning robot controller is divided into several logic parts. The processing of each logic part shall be in accordance with certain principles, at the same time, also follows general principles of simulation analysis of cleaning robot controller, which is analyzed in detail.

\subsection{Overall framework}

The framework of cleaning robot can be divided into data analysis, auxiliary and path planning. Data analysis include data collection, data loading, data analysis and data processing. Data 
collection and processing include robot motion data and environment perception data. In order to better conduct data collection and analysis, relevant personnel shall set algorithm inside cleaning robot, which can simplify analysis process of data to allow robot to response to data more rapidly and better.

Auxiliary part can help robot accomplish more complex content, including auxiliary design of a certain motion path, analysis of motion specific problems, garbage processing and so on.

Motion track is an important part in the operation of cleaning robot, and path planning include motion path planning and joint space path planning. Motion path planning include cleaning route planning and obstacle avoidance planning. Cleaning route planning shall satisfy two characteristics. First of all, low route repeatability. The repeatability of route will consume extra time and energy sources of robot, which contradicts the performance requirements of cleaning robot. Secondly, low cleaning loopholes. One feature of evaluating the performance of cleaning robot is cleaning loopholes. The design of cleaning robot is to ensure low cleaning loopholes while guaranteeing low route repeatability, which requires the flexibility of system algorithm of cleaning robot to deal with different situations. Obstacle avoidance planning shall satisfy two characteristics. First of all, pre-analysis. In many cases, the collision between cleaning robot and the material object will cause some damage to the cleaning robot and material object. In order to avoid this kind of problem, in programming, it is necessary to pay attention to the pre-analysis and its programming mechanism. The pre-analysis means that the robot can avoid the impending collision event according to the actual situation. The specific avoidance methods include slowing down the running speed and changing the motion path in time. Both methods have certain advantages and disadvantages, and the user can select an appropriate method according to their own use requirements, or combine two methods to further improve the intelligence of the cleaning robot. Secondly, high avoidance accuracy. Avoidance costs a lot sometimes. For example, changes of route can bring consumption of time. The increasing of avoidance accuracy can better solve problems to improve the function of cleaning robot.

\subsection{Matlab simulation framework}

Matlab is a software with outstanding performance and a whole set of system process, which can better solve problems in actual operation. The content of Matlab simulation framework is described specifically.

First of all, graphical user interface. Simulation with the application of graphical user interface has more advantages than simulation with direct codes, more visualized and easier to understand, with more operating space and lower use difficulty. The simulation with graphical user interface can to great extent improve the efficiency of simulation, and operator will not deal with traditional complex operating codes, which can strengthen the interactivity and usability of program.

At the same time, simulation with graphical user interface can better accomplish some operations, like motion control, which is more visualized to allow relevant personnel understand.

Secondly, it can better draw the working device, working enveloping space of robot, and the button of current working space of robot.

Thirdly, it can pre-draw the attitude angle and position of target. The simulator only needs to select a point in the graphics window and give the information of the point as feedback to the system to pre-draw the attitude angle and position of the target. In addition, inputting corresponding information in the corresponding input box can also pre-draw the attitude angle and position of the target.

Fourthly, it has two inverse kinematics methods and simulation operating modes. Users can conduct method setting or select corresponding method for method setting based on their use requirements, which increases the flexibility of robot simulation, applied to more complex situations.

Fifthly, it can conduct method of robot's kinematics and inverse kinematics. After clicking the corresponding button, users can set the relevant information. After information setting, the system automatically feeds back the relevant content according to the content of information setting. 
Sixthly, visual simulation. Visual simulation is consistent with simulation requirements of users. With visual simulation, users can better observe simulation results. Besides, visual simulation can also update data dynamically, which is in accordance with real life.

Seventhly, it can give prompts of wrong information combined with actual situation. There are maybe some errors in simulation, which has great impact on simulation results, including blind position and blind angle. Whereas, in simulation, the system can capture these contents automatically and feed back wrong contents.

Eighthly, high compatibility. Sometimes, the function that Matlab provides to users is limited. In this case, users may need to combine other development tools. The current Matlab software is better compatible with other software. Users can import other people's code or plug-ins into their own programs in a few simple steps, which further simplifies the simulation process.

\section{Simulation Procedures}

In the application of Matlab software, it is necessary for relevant personnel to conduct the simulation of cleaning robot controller according to procedures, which is established based on the full understanding by operator on the second part of this paper.

\subsection{The setting of operating environment}

Matlab provides strong function, suitable for the development of more tools, therefore, Matlab sets certain attribute setting interface. In simulation analysis, the setting of attribute interface in advance is very important. The better operational environment of Matlab can better satisfy use requirements of users.

\subsection{The model design of cleaning robot}

The model design of cleaning robot is an important part in simulation analysis. In application model, users can independently built their own model, and also can design model based on certain models. Whereas, the latter has better application performance, which can better avoid errors of users and provide more application basis for users. After the construction of model, relevant personnel is required to analyze further the model based on model parameters. As for the complex unit, users can directly refer to source program of Matlab to better understand the model.

\subsection{The data loading}

After users press the start button, some of the robot's program interfaces are automatically opened. In this case, users can manually input the data. After the data inputting, the data is slowly loaded into the simulation model of cleaning robot controller. At the loading, the data is connected to some of the components in the simulation model based on relevant operations in the program interface. This process is also called initialization of the simulation. It is worth noting that the input of data is sometimes complicated. At this time, the operator can write some data into local file, and the local file can be applied during the initialization process to complete the initialization of the simulation model.

\subsection{The Matlab programming}

In programming with Matlab, users can make full use of some functions provided by graphical interface to simplify programming procedures, which is detailed. First of all, build a coordinate system. It is very important to establish a coordinate system related to the cleaning robot connecting rod, which is the basis for performing the cleaning robot motion path analysis. Secondly, perform the solution of kinematic equation and obtain inverse kinematics. Thirdly, build the model related to cleaning robot controller, and perform mathematical calculation of kinematics and inverse kinematics after the establishment of model. Fourthly, generate corresponding path according to actual situations. It is necessary to give considerations to coordination of path in the process. Fifthly, perform the design of relevant coordinate based on actual operating requirements. The coordinate system established in the first step is sometimes not conducive to the analysis of the whole. In this 
case, users can design the relevant coordinate system according to actual operating requirements, which can better reduce the difficulty of users. Sixthly, accomplish interface function and corresponding program control. Seventhly, conduct the simulation analysis. After confirming that the above operations are correct, users can perform the model instantiation.

\subsection{The interactive simulation}

During the simulation, the system will provide users with certain operational options based on the actual situation of the simulation. Users need to select the content of the option according to their own purpose. For example, in the "Path Planning", users can select "Joint Space" or other content. In the interactive simulation, users need to record the running characteristics of the simulation and analyze the running characteristics of the simulation as much as possible. In addition, users also need to have a full understanding of the errors in the simulation process, which is the basis for ensuring that the simulation can better meet users' needs and further improve the model of the cleaning robot controller.

\subsection{The analysis of simulation results}

There may be unexpected emergency happening during the simulation. In this case, the user needs to analyze the results of the simulation and find the root cause of the problem based on the results of the simulation. Only by repeating this step can the user further optimize the performance of the cleaning robot controller. The analysis of simulation results is described in detail. First of all, the functionality. The analysis of functionality refers to the completion of the simulation on the function required of cleaning robot controller, which includes the rationality of the route planning mentioned above and the rationality and accuracy of the avoidance route planning. Secondly, the sufficient simulation analysis. Whether the simulation analysis is sufficient includes several aspects, such as whether the user has better processed and analyzed multiple simulation contents. It is very important to be able to process and analyze multiple simulation contents because the work required of cleaning robot controller is quite complicated. Only when the cleaning robot controller analyzes multiple situations can it be more suitable for a complex environment. Thirdly, the consistency between simulation analysis and actual situations. The simulation analysis of cleaning robot is a simulation of the actual society. The specific operation principle is that the software provides functions and creates a real-world simulation environment combined with users' programming. In this simulation environment, cleaning robot controller is also one of the simulation contents. As a part of real time simulation, the simulation may have a big conflict with the actual situation, which is not conducive to the further development of cleaning robot simulation. Therefore, relevant personnel must pay attention to the consistency test of the simulation analysis of cleaning robot and the actual situation.

\section{Conclusion}

This paper briefly discusses the simulation design of cleaning robot controller based on Matlab. The application of Matlab is beneficial to solve many problems in simulation, and it can also better coordinate the problems of students' use requirements and teaching resources. In the overall framework of cleaning robot controller, users need to pay attention to the two parts mentioned above, the overall framework and the Matlab simulation framework. In applying Matlab to the actual simulation of cleaning robot controller, users need to pay sufficient attention to the complicated operation procedures and some operation details mentioned above, which lays a foundation for better operation of simulation.

\section{Acknowledgement}

Fund project: This paper comes from science and technology project of Jiangxi Education Department. Project Name: Design and Simulation of Intelligent Controller of Cleaning Robot. Project No. 161564 


\section{References}

[1] Yin Xincheng, Hu Yong. Research on Robot GUI Simulation Platform Design Based on MATLAB [J]. Bulletin of Science and Technology, 2018(1).

[2] Ye Renping, Zeng Dehuai. Research on Jointed Simulation of Robot Arm Motion Control Based on Adams and Matlab [J]. Modern Manufacturing Engineering, 2017(6):56-61.

[3] Shi Danni. Design and Simulation of Autonomous Bicycle Control System [D]. China University of Geosciences (Beijing), 2017.

[4] Cai Mingxue. Design of Obstacle Avoidance System of Robot Based on Fuzzy Control [J]. Intelligent Robot, 2017(3):73-75. 